\title{
Modellazione efficiente agli elementi finiti per l'analisi a collasso di strutture incollate complesse
}

\author{
D. Castagnetti, A. Spaggiari, E. Dragoni \\ Università di Modena e Reggio Emilia, Dipartimento di Scienze e Metodi dell'Ingegneria, via Amendola, 2 -42100 Reggio \\ andrea.spaggiari@unimore.it
}

\begin{abstract}
RIASSUNTO. Il lavoro verifica l'applicabilità di un modello semplificato agli elementi finiti per l'analisi a collasso post elastico di strutture incollate complesse in parete sottile. Al fine di superare le limitazioni dei modelli di letteratura come l'uso di elementi speciali, il lavoro sfrutta un modello ridotto già presentato dagli autori in campo elastico. Tale modello è basato sulla rappresentazione degli aderendi mediante elementi semistrutturali (piastre o gusci) e dell'adesivo per mezzo di speciali elementi coesivi. La continuità strutturale tra aderendi e adesivo è ottenuta mediante vincoli interni (tied mesh) che accomunano i gradi di libertà dei nodi mutuamente affacciati di aderendi ed adesivo.

La struttura analizzata è un simulacro di incollaggio industriale e produce nella strato adesivo una sollecitazione complessa, analizzabile solo con modelli numerici. Si considera una struttura tubolare in parete sottile a sezione quadrata, fatta di due spezzoni posti testa a testa e incollati con fazzoletti di lamiera sui quattro lati. La struttura è sottoposta a flessione a tre punti fino al cedimento e la zona incollata posta disassata rispetto al punto di applicazione del carico riceve una sollecitazione indiretta. I risultati dell'analisi FEM, confrontati direttamente con le curve sperimentali forza-spostamento, evidenziano una buona accuratezza del metodo, in termini di rigidezza, forza massima e comportamento post elastico della struttura, accompagnati da ridotte dimensioni del modello e tempi di calcolo molto contenuti. Grazie a questi vantaggi, la procedura si presta ad effettuare l'analisi di strutture incollate complesse, altrimenti ingestibili se affrontate con una modellazione agli elementi finiti tradizionale.
\end{abstract}

ABSTRACT. The paper deals with the application of an efficient finite element (FE) model for the failure analysis of bonded structures. Aim of the work is to assess the accuracy and applicability of the computational model in the prediction of the post-elastic response of large and complex bonded structures. In order to overcome the limitations encountered in the technical literature, such as the use of special elements, the present work assesses the applicability of a reduced computational method, previously presented by the authors. The method is based on standard modeling tools, which are available in most of commercial FE packages. The method describes the adherends by semi-structural elements (plates or shells), and the adhesive by means of a single layer of cohesive elements.

This work applies the proposed reduced method to a complex, industrial-like, structure. A square thin-walled beam is considered, made of two different portions joined head to head by overlapping thin plates on each side. The beam is loaded by a three point bending fixture up to failure which originates an indirect, complex stress field on the bonded region.

The benchmark for the computational analyses are the force-displacement curves obtained by experimental tests on two different geometries. The comparison with the experimental data shows a good accuracy of the proposed method in terms of structure stiffness, maximum load and post-elastic behaviour up to the collapse of the structure. The numerical precision and the computational speed make the proposed method very useful for the efficient analysis of complex bonded structure, both for research and industrial purposes.

Parole Chiave. Metodi numerici efficienti, analisi a collasso, costruzioni incollate 


\section{INTRODUZIONE}

I

1 lavoro riguarda l'applicazione di un modello efficiente agli elementi finiti (EF), precedentemente verificato dagli autori in campo elastico, per l'analisi a collasso di strutture incollate. L'obiettivo del lavoro è di valutare l'accuratezza e l'applicabilità del modello computazionale nella previsione della risposta post-elastica di strutture incollate complesse di dimensioni anche elevate utilizzando strumenti computazionali standard.

La motivazione della ricerca risiede nel fatto che l'applicazione industriale delle giunzioni strutturali incollate è legata allo sviluppo di metodi di calcolo semplici, veloci e accurati per la previsione della loro resistenza meccanica.

In letteratura si ritrovano numerosi metodi agli elementi finiti per l'analisi delle giunzioni incollate [1-10]. Molti di questi metodi sono basati su elementi speciali per descrivere lo strato adesivo o la zona di sovrapposizione. I principali svantaggi di questi metodi risiedono nel fatto che gli elementi speciali da essi impiegati sono difficili da implementare nei software agli elementi finiti commerciali impiegati nell'ambito industriale e il loro uso è confinato ad applicazioni di ricerca. In lavori recenti, invece, i metodi più comunemente impiegati adottano approcci basati sulla meccanica della frattura [11-14]. In questo caso, i criteri di cedimento impiegati, richiedono dati che difficilmente sono forniti dal produttore dell'adesivo e devono quindi essere ottenuti sperimentalmente.

Per superare queste limitazioni, il presente lavoro approfondisce l'analisi di un metodo computazionale semplificato, già presentato dagli autori in [15], per l'analisi di giunzioni strutturali in parete sottile. Il metodo è basato su strumenti di modellazione standard e su elementi finiti comuni, implementati nella maggior parte dei software di calcolo commerciali. Il metodo descrive gli aderendi mediante elementi semi-strutturali (piastre o gusci), l'adesivo mediante un singolo strato di elementi solidi e ricorre a vincoli cinematici interni per riprodurre la continuità strutturale. In [15] si è dimostrata l'efficienza e l'accuratezza del modello ridotto nel calcolare la distribuzione delle tensioni elastiche lungo il piano medio dello strato adesivo per parecchie geometrie 2D e 3D. Successivamente, gli autori hanno esteso il metodo in campo postelastico [17, 22] adottando il semplice criterio di cedimento alle tensioni regolarizzate proposto in $[16,20]$ ed ottenendo risultati incoraggianti.

Questo lavoro estende l'applicazione del metodo ridotto ad una trave tubolare, composta da due tratti diseguali incollati testa a testa mediante sovrapposizione di lamierini. La trave è caricata a flessione su tre punti fino a completo collasso ed origina uno stato tensionale complesso sulla zona di incollaggio. Si è implementato un criterio di cedimento secondo l'approccio della zona coesiva come proposto in [21] in modo da unire accuratezza del modello e velocità di calcolo. L'elemento di confronto per le analisi computazionali è rappresentato dalle curve forza-spostamento ottenute da prove sperimentali su giunzioni tubolari incollate con la stessa geometria di quelle studiate numericamente.

L'originalità del lavoro consiste nella semplicità degli strumenti computazionali proposti, basati su opzioni standard di modellazione disponibili in ogni pacchetto di calcolo agli elementi finiti commerciale. Ne deriva un metodo generale e di facile impiego, caratterizzato da una forte riduzione del costo computazionale (occupazione di memoria e tempo di calcolo), conseguente alla minimizzazione dei gradi di libertà del modello. Semplicità, generalità ed efficienza fanno del metodo proposto un valido strumento industriale per simulare il comportamento meccanico di strutture incollate grandi e complesse.

\section{MATERIALI E METODI}

I 1 lavoro è diviso in due fasi: analisi computazionali e prove sperimentali, queste ultime ancora in fase esplorativa e condotte solo su due tipi di geometria. E' stata considerata una struttura trabeiforme (Fig. 1), costituita da due spezzoni di tubo quadro uniti da piastrine di collegamento incollate per sovrapposizione semplice su ciascun lato. La struttura viene caricata a flessione su tre punti. Essendo la zona di unione lontana dalla mezzeria, nell'adesivo delle giunzioni si sviluppa uno stato di sollecitazione indiretto e complesso. La struttura, di semplice realizzazione, è più un simulacro di una struttura reale incollata che una semplice provino di laboratorio e costituisce un buon banco di prova per il metodo proposto. Le prove sia computazionali che sperimentali sono stato condotte fino al collasso della struttura.

\section{Prove sperimentali}

Le prove sperimentali svolte sono state di carattere esplorativo per valutare quali e quanti fattori considerare in una futura serie di prove sistematiche. La Fig. 1 rappresenta schematicamente la geometria considerata per la giunzione. In Fig. 1-a si 
riportano le dimensioni della struttura incollata mentre in Fig. 1-b si riporta la geometria della struttura tubolare semplice, senza giunzione. Quest'ultima ha la funzione di riferimento per valutare l'influenza globale della giunzione sulla resistenza della struttura.

Sono state considerate due differenti dimensioni degli aderendi per ognuna delle due configurazioni. In Tabella 1 sono riportate le dimensioni e i materiali scelti.

Gli aderendi sono tubi quadri in acciaio da costruzione Fe510 e l'adesivo impiegato è un epossidico bi-componente ad alta resistenza (Henkel 9466 [18]). La Tabella 1 raccoglie le proprietà elastiche degli aderendi e dell'adesivo mentre il loro comportamento post-elastico è descritto dalle curve di Fig. 2a e 2b. La larghezza delle piastre di collegamento è di $25 \mathrm{~mm}$ e lo spessore dello strato adesivo è stato ottenuto portando a contatto le parti e lo si è ipotizzato pari a $0.05 \mathrm{~mm}$, dovuto solo alla rugosità degli aderendi.

Gli aderendi sono stati preparati, prima di incollarli, attraverso una levigazione meccanica con carta abrasiva (grana 200) e successivamente puliti con il solvente sgrassatore Henkel Loctite 7063 [19], per garantire una migliore adesione substratoadesivo.

Le prove sperimentali sono state svolte ad una velocità costante della traversa di $60 \mathrm{~mm} / \mathrm{s}$ fino al collasso completo del giunto. La macchina di prova usata è una MTS MINI Bionix 858, servo idraulica, con capacita assiale di $25 \mathrm{KN}$.

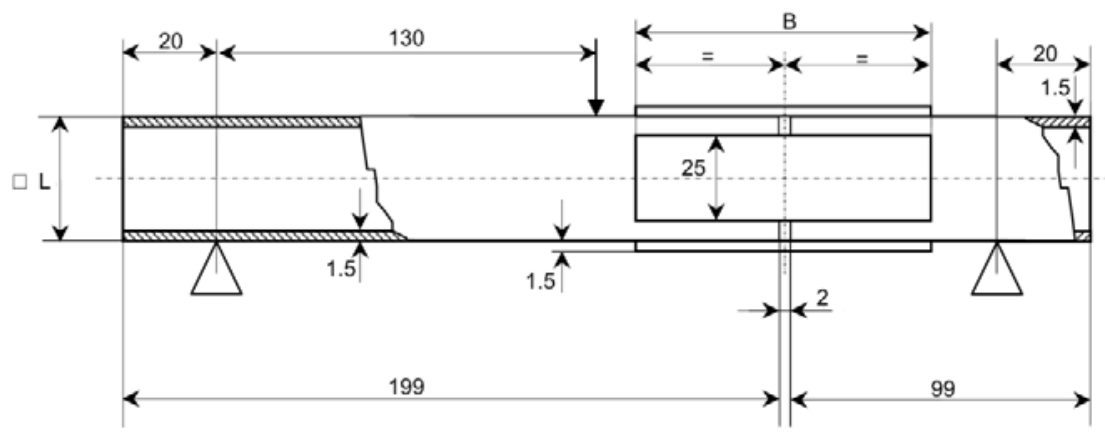

(a)

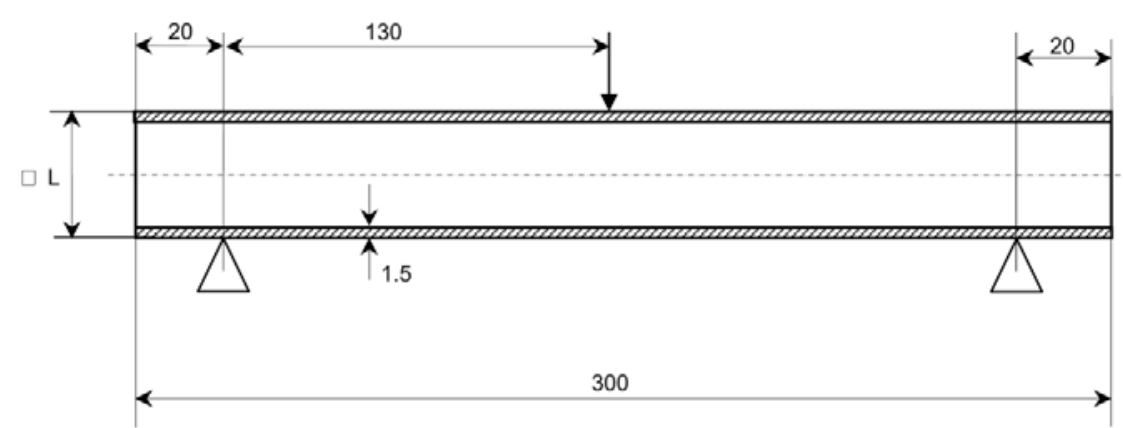

(b)

Figura 1: Schema della struttura tubolare incollata (a) e del tubo quadro integro (b)

\begin{tabular}{ccc}
\hline & & Geometria \\
L $(\mathrm{mm})$ & 25 & 40 \\
B $(\mathrm{mm})$ & 50 & 0.05 \\
Spessore adesivo $(\mathrm{mm})$ & \multicolumn{2}{c}{ Materiali } \\
& Aderendi & Adesivo \\
& Acciaio & Henkel Loctite 9466 \\
Modulo di Young $(\mathrm{MPa})$ & 206.000 & 1718 \\
Coefficiente di Poisson & 0.3 & 0.3 \\
Tensione elastica massima $(\mathrm{MPa})$ & 500 & 60 \\
\hline
\end{tabular}

Tabella 1: Variabili geometriche e proprietà meccaniche materiali. 


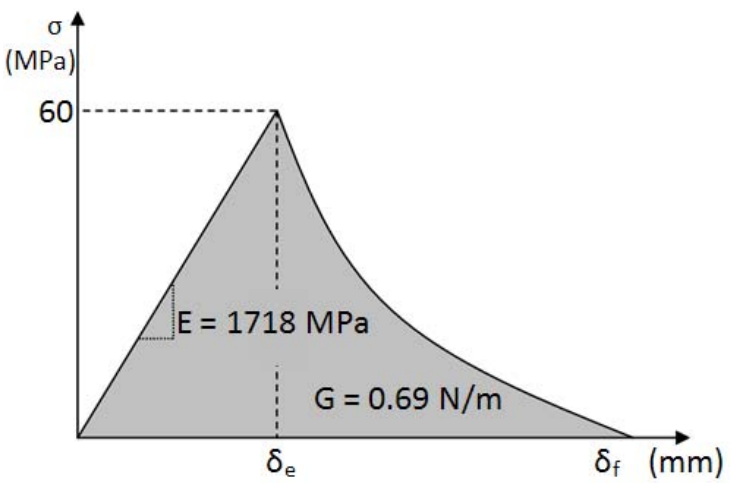

(a)

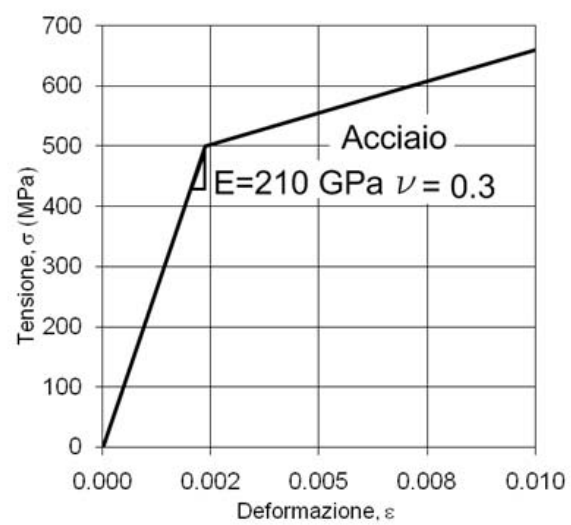

(b)

Figura 2: Legame coesivo adesivo (a) e legame costitutivo acciaio elastoplastico (b)

\section{Analisi computarionale}

Lo scopo dell'analisi computazionale è di ottenere la curva forza spostamento fino al collasso completo, permettendo un confronto diretto con i risultati sperimentali.

Il modello computazionale è stato sviluppato in forma tridimensionale sia per la struttura tubolare incollata sia la struttura integra. Gli aderendi sono stati descritti mediante elementi semi-strutturali di tipo piastra (shell) collocati sulle superfici medie delle pareti del tubo. Lo strato adesivo è descritto mediante un singolo strato di elementi coesivi solidi. La modellazione degli aderendi mediante elementi strutturali determina una discontinuità virtuale tra aderendi ed adesivo. Per ripristinare il collegamento, si impiegano vincoli cinematici interni che rendono uguali i gradi di libertà corrispondenti delle parti vincolate. Sia gli aderendi sia l'adesivo sono stati modellati per mezzo di elementi lineari ad integrazione ridotta, aventi forma quadrata. La dimensione della mesh sull'aderendo è pari alla distanza dei piani medi degli aderendi, mentre l'adesivo è discretizzato con elementi aventi lato pari a un quarto della distanza dei piani medi degli aderendi. Questa scelta, derivante da osservazioni effettuate in lavori precedenti degli autori, ha fornito un buon compromesso tra precisione dei risultati e tempi di calcolo ragionevoli [22].

I modelli computazionali sono stati sviluppati per tutte le configurazioni esaminate sperimentalmente e sono stati implementati mediante il solutore esplicito del software agli elementi finiti ABAQUS 6.8 [23].

Gli aderendi sono stati modellati con un semplice legame elasto-plastico incrudente bilineare (Fig. 2a), mentre l'adesivo è stato descritto mediante il modello di zona coesiva di Fig. 2b. I valori di snervamento degli aderendi sono stati ottenuti sulla base dei dati forniti dal produttore dei tubi mentre i parametri che governano l'andamento della zona coesiva (tensione massima $=60 \mathrm{MPa}$, energia di frattura $=0.69 \mathrm{~N} / \mathrm{m}$ ) sono stati ricavati da lavori di letteratura [24] riguardanti il medesimo adesivo. Il criterio scelto prevede che al raggiungimento del limite elastico, in modo I, II, III l'adesivo perda progressivamente le sue proprietà meccaniche con legge esponenziale.

$\mathrm{Al}$ modello agli elementi finiti è stato applicata centralmente una velocità di spostamento di $150 \mathrm{~mm} / \mathrm{s}$, di poco superiore a quella sperimentale, e l'opzione di scalatura della massa, tecniche che consentono di ridurre i tempi di analisi, senza pregiudicarne i risultati. Dalle analisi si è ricavato il carico di reazione della struttura fino al suo cedimento. Tutti i modelli sono stati risolti mediante un processore Intel Core Duo Mobile T7200.

\section{RISULTATI}

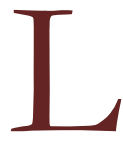

a Fig. 3 raccoglie i risultati sperimentali, in termini di diagramma forza-spostamento, per le configurazioni considerate. La Fig 3a si riferisce al tubo di lato $25 \mathrm{~mm}$ mentre la Fig $3 \mathrm{~b}$ è relativa al giunto di lato $40 \mathrm{~mm}$.

In ogni diagramma è rappresentata sia la curva prodotta dalla trave incollata (linea nera sottile) che la curva generata dall'analogo tubolare integro (linea grigia spessa). 
Per ogni prova sperimentale si è effettuata la relativa simulazione agli elementi finiti. In Fig. 4 si riporta il confronto tra la simulazione numerica (linea nera spessa) e la prova sperimentale (curva grigia sottile) per quanto riguarda il tubo integro, mentre in Fig. 5 si riporta il confronto sul tubo incollato.

In Tab. 2 sono riportati i tempi di calcolo per le prove numeriche effettuate con una macchina di fascia media, processore Intel T7200 1.99GHz, Ram 2Gb.

In Fig. 6 si mostra invece il confronto tra le immagini sperimentali delle prove di flessione e le relative simulazioni agli elementi finiti.

In Fig 6a si riporta la prova sperimentale sul tubo di lato $25 \mathrm{~mm}$, Fig 6b si riporta la prova sperimentale sul tubo di lato $40 \mathrm{~mm}$, in Fig. 6c la simulazione del tubo di lato $25 \mathrm{~mm}$ e in Fig. 6d la simulazione del tubo di lato $40 \mathrm{~mm}$.

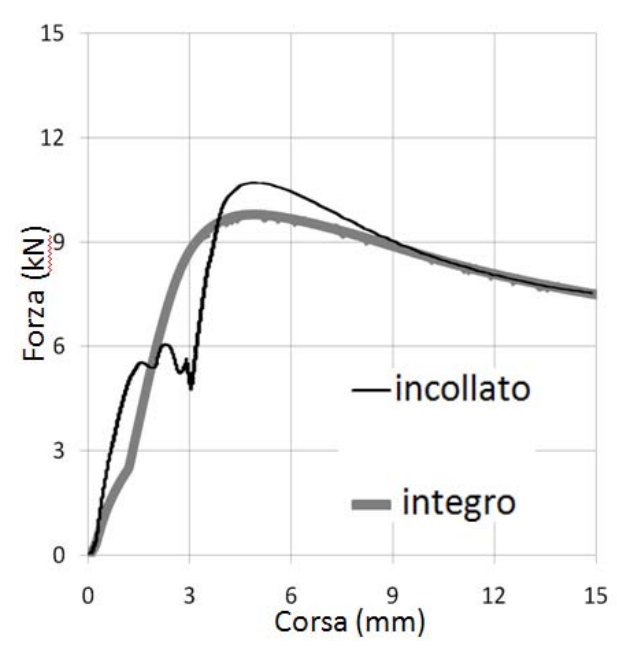

(a)

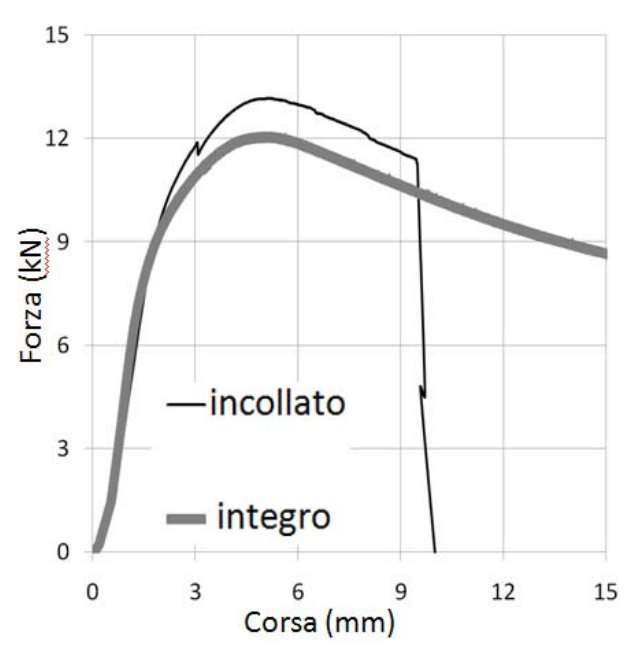

(b)

Figura 3: Curve sperimentali forza - corsa del tubo lato $25 \mathrm{~mm}$ (a) e lato $40 \mathrm{~mm}$ (b).

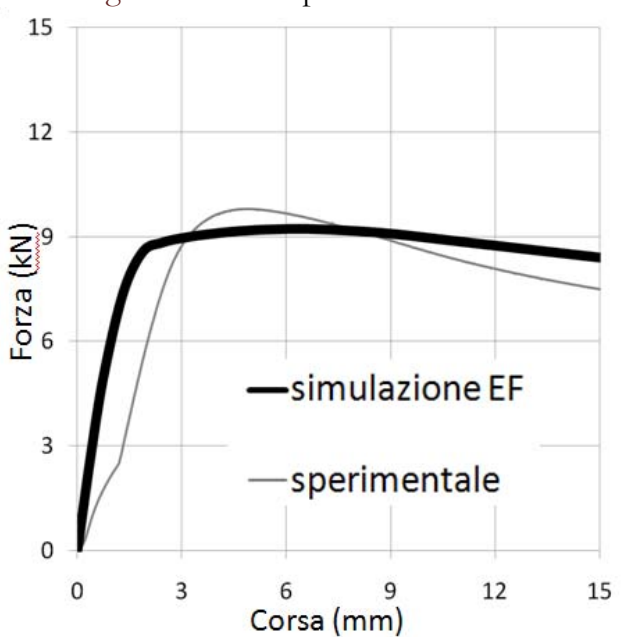

(a)

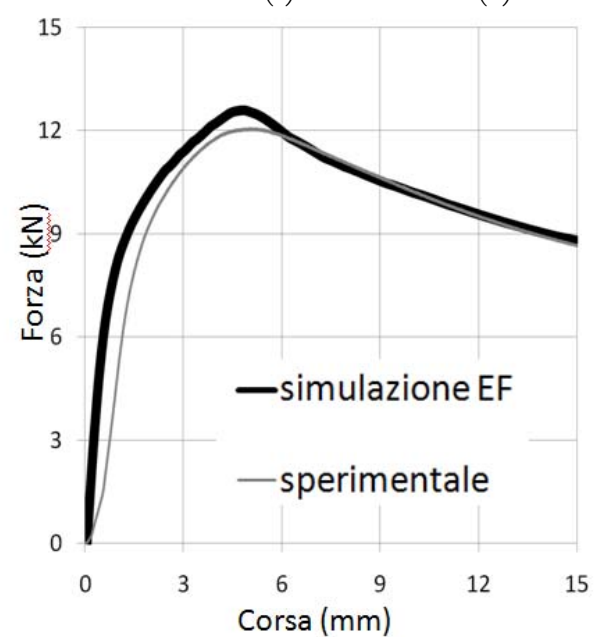

(b)

Figura 4: Confronto numerico-sperimentale tubo integro lato $25 \mathrm{~mm}$ (a) e lato $40 \mathrm{~mm}$ (b).

\begin{tabular}{ccc}
\hline & \multicolumn{2}{c}{ Lato Tubo (mm) } \\
Tipo tubo & $\mathbf{2 5}$ & $\mathbf{4 0}$ \\
Integro & 96.5 & 340.3 \\
Incollato & 5085 & 5438 \\
\hline
\end{tabular}

Tabella 2: Tempi di analisi (s) - Processore Intel T7200 1.99GHz, Ram 2Gb. 


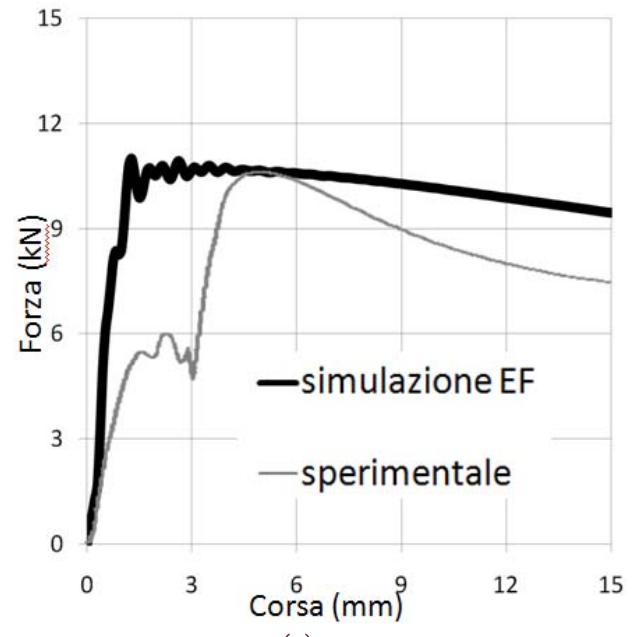

(a)

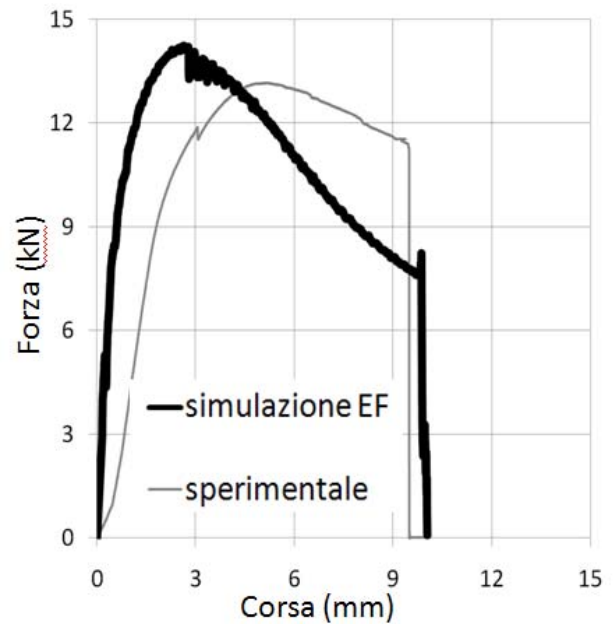

(b)

Figura 5: Confronto numerico-sperimentale tubo incollato lato $25 \mathrm{~mm}$ (a) e lato $40 \mathrm{~mm}$ (b).

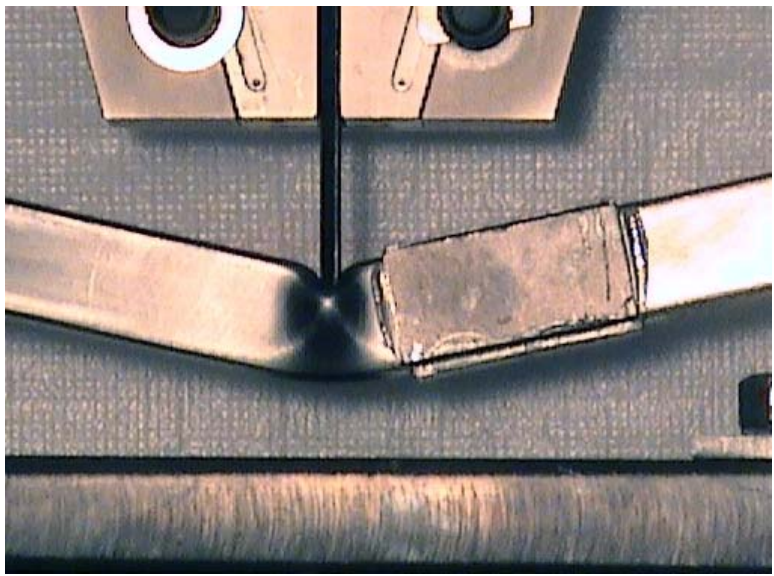

(a)

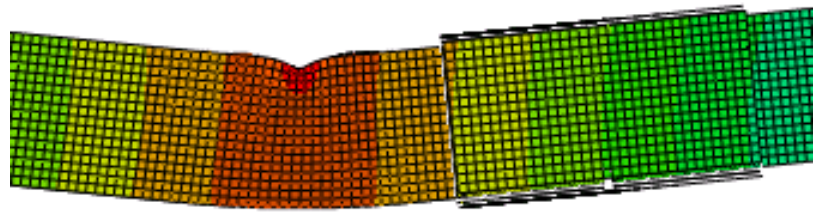

(c)

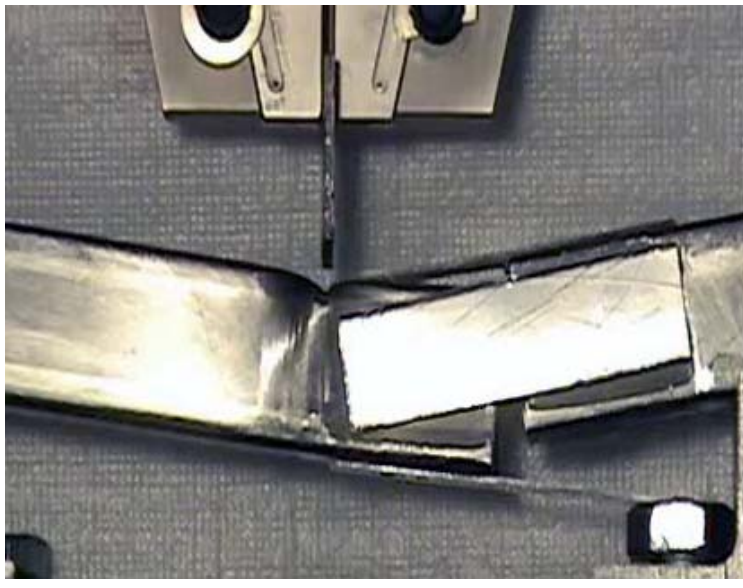

(b)

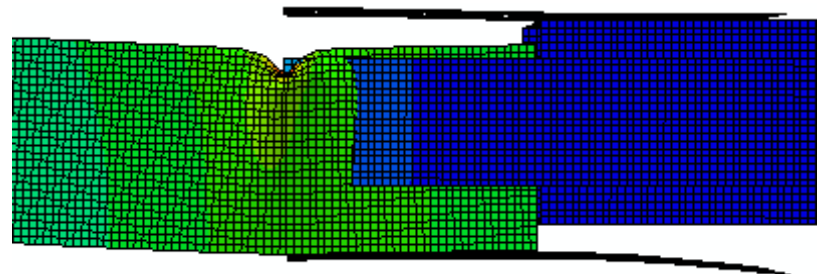

(d)

Figura 6: Prova sperimentale su tubo incollato lato $25 \mathrm{~mm}$ (a) e lato $40 \mathrm{~mm}$ (b). Simulazione numerica (mappa degli spostamenti) del tubo incollato lato $25 \mathrm{~mm}$ (c) e lato $40 \mathrm{~mm}$ (d).

\section{DISCUSSIONE}

\section{Prove sperimentali}

T n primo luogo si nota dalle curve sperimentali riportate in Fig. 3 che il carico sostenuto dal tubo quadro integro è inferiore a quello dello stesso tubo tagliato e incollato. Questo comportamento si riscontra per entrambe le geometrie considerate. Ciò è spiegabile in parte poiché lo spessore di parete nella zona incollata sostanzialmente raddoppia aumentando quindi il modulo di resistenza della sezione, ma è anche indice che l'adesivo trasferisce il 
completamente ed efficientemente il carico. Da questo punto di vista è importante notare che in ambedue le prove lo snervamento dell'acciaio avviene prima del cedimento dell'adesivo (Fig. 3). Nel caso del tubo di lato 25mm (Fig. 5a), l'adesivo non presenta cedimenti catastrofici e la prova è stata terminata quindi per il raggiungimento della corsa massima disponibile. L'oscillazione nel tratto iniziale è forse imputabile a una microfrattura nella zona incollata, in quanto durante l'esecuzione della prova si sono rilevati rumori che sembravano indicare una nascente cricca nell'adesivo. Questo cedimento probabilmente è occorso in una zona incollata non critica, forse a causa di inclusioni di aria, e la prova è quindi continuata senza cedimenti della struttura fino alla completa plasticizzazione del tubo.

Dal diagramma di Fig. 3b si osserva inoltre che la curva sperimentale del tubo incollato di lato $40 \mathrm{~mm}$ pur avendo un carico massimo maggiore del tubo integro ha un cedimento prematuro con rottura catastrofica della struttura.

Non avendo effettuato ripetizioni della prova questo non è un dato statisticamente rilevante ma è possibile motivare la differenza a fronte di alcune considerazioni sulla differente geometria delle strutture.

In Fig. 6-a si nota come nella prova di flessione nel tubo di lato $25 \mathrm{~mm}$ la cerniera plastica, originata sugli aderendi dal carico flessionale, è sufficientemente lontana dalla zona incollata e non influisce quindi sulla zona di incollaggio. Nel caso della prova di Fig. 6-b, invece, l'inizio del tratto incollato è molto più vicino alla cerniera plastica. Vi è quindi una influenza diretta della deformazione prodotta nell'aderendo dalla cerniera plastica sulla zona di incollaggio. Questo può determinare una possibile causa del cedimento prematuro della struttura. Un'altra differenza rispetto alla struttura incollata di lato $25 \mathrm{~mm}$ è che la piastra incollata sul tubo di lato $40 \mathrm{~mm}$ è più stretta rispetto al lato del tubo, infatti il rapporto tra la piastra e il tubo da $40 \mathrm{~mm}$ è 0.625 mentre è 1 per il tubo di lato $25 \mathrm{~mm}$. Questo significa che il tubo di lato $25 \mathrm{~mm}$ è più rinforzato rispetto all'altro e questo giustifica la sua maggiore capacità di sostenere carico.

\section{Confronto numerico - sperimentale}

In Fig. 4 si evidenzia un buon accordo tra le curve sperimentali e la simulazione agli elementi finiti del tubo integro per tutte le configurazioni esaminate.

La differenza maggiore si ha nel tratto elastico ed è spiegabile in quanto l'inizio della prova sperimentale è affetto da un assestamento della struttura alla prima applicazione del carico e ciò comporta una discrepanza tra le curve. Inoltre la mesh sugli aderendi è abbastanza rada il che irrigidisce ulteriormente la struttura. La pendenza del tratto elastico, però, è comparabile e l'errore sulla rigidezza molto contenuto. Per quanto riguarda la resistenza della struttura l'errore sulla forza massima è inferiore a $\pm 7 \%$ e anche il tratto post elastico viene ben colto dalla simulazione.

La Fig. 5 mostra un discreto accordo tra le curve sperimentali (linee grigie sottili) e la simulazione agli elementi finiti del tubo incollato (linee nere spesse).

In primo luogo si rileva che le oscillazioni presenti nelle curve numeriche sono dovute alla modalità di simulazione esplicita che è stata adottata e pertanto non vanno considerate. E' stato anche effettuata una scalatura della massa della struttura che aumenta questo effetto, ma consente di abbreviare in maniera consistente il tempo di analisi.

In Fig $5 \mathrm{a}$ si mostra il confronto per il tubo di lato $25 \mathrm{~mm}$. Non si è verificato il collasso del tubo e quindi non si hanno informazioni precise né sulla energia assorbita dallo strato adesivo né sull'istante di collasso, ma si registra solamente che la giunzione è sufficientemente resistente per portare a snervamento completo il tubo.

La simulazione evidenzia una rigidezza comparabile a quella della prova di flessione e una forza massima di $10.98 \mathrm{kN}$, di poco superiore al dato sperimentale di $9.8 \mathrm{kN}$, con un errore del $10 \%$. Il tratto post elastico della simulazione decresce in maniera meno accentuata rispetto alla prova reale ma ciò è probabilmente imputabile al modello di materiale bilineare incrudente usato per gli aderendi.

In Fig 5b, invece, si osserva che, per il tubo incollato di lato $40 \mathrm{~mm}$ la risposta della simulazione è più rigida nel primo tratto elastico a causa sia della cedevolezza della attrezzatura sperimentale, ma anche della mesh rada sull'aderendo che irrigidisce ulteriormente la struttura.

In Fig. 6c si riporta la mappa degli spostamenti della simulazione sul tubo di lato $25 \mathrm{~mm}$, in ottimo accordo con la prova sperimentale di Fig. 6a, mentre in Fig. 6d si mostra la mappa degli spostamenti del tubo di lato 40mm all'istante in cui avviene il cedimento completo dell'adesivo. E' interessante notare il buon accordo tra le deformate sperimentali e quelle simulate, anche se la Fig. $6 \mathrm{~d}$ presenta una differenza sostanziale con la prova sperimentale di Fig. $6 \mathrm{~b}$ dovuta alla mancanza della forza di gravità. Infatti nella prova sperimentale i due spezzoni di tubo, dopo il cedimento dell'adesivo si adagiano 
l'uno sull'altro, mentre nella simulazione la rottura dello strato adesivo crea un effetto di ritorno elastico per cui i due spezzoni tendono ad allontanarsi.

La forza massima sviluppata dalla simulazione nel caso del tubo di lato $40 \mathrm{~mm}$ è $14.2 \mathrm{kN}$ contro i $13.2 \mathrm{kN}$ della prova sperimentale con un errore del $7.5 \%$. La previsione computazionale del tratto post-elastico mostra una discesa prematura del carico sopportato dalla struttura mentre prevede in ottimo accordo con la curva sperimentale con esattezza il livello di inflessione che porta a completo collasso la struttura. Il tempo necessario all'analisi, di una costruzione incollata di questo genere mostrato in Tab. 2, si attesta sui 5000 secondi. Ciò rende il metodo proposto valido anche per l'analisi costruzioni di dimensioni maggiori, che si possono facilmente incontrare in un contesto industriale, senza compromettere la precisione dei risultati che si mantiene buona, essendo l'errore sempre inferiore al $10 \%$.

\section{CONCLUSIONI}

I 1 lavoro mostra l'applicabilità di un modello semplificato agli elementi finiti per l'analisi a collasso di strutture incollate complesse. Il modello è applicato ad una struttura tubolare incollata ed è confrontato direttamente con le prime prove sperimentali esplorative realizzate. Il modello è basato sulla rappresentazione degli aderendi mediante elementi shell e dell'adesivo per mezzo di speciali elementi coesivi. I nodi corrispondenti di aderendi ed adesivo sono collegati da vincoli interni tipo tied-mesh per ripristinare virtualmente la continuità fisica della giunzione. Il confronto con i risultati sperimentali evidenzia una buona accuratezza del metodo sia in termini di forza massima prevista sia di comportamento post-elastico. In particolare si hanno stime sulla forza massima sopportata dal giunto con errori inferiori al 10\%, una buona previsione dell'istante di collasso (ove esso si verifica) e una discreta previsione della rigidezza.

La buona precisione numerica ed il ridotto peso computazionale (bassa occupazione di memoria e bassi tempi di calcolo) rendono il metodo proposto particolarmente adatto per l'analisi efficiente di costruzioni incollate complesse di interesse industriale.

\section{BIBLIOGRAFIA}

[1] B. N. Rao, Y. V. K. S. Rao, S. Yadagiri, Fibre Science and Technology, 17 (1982) 77.

[2] J. N. Reddy, S.Roy, Int. J. Non-Linear Mechanics, 23 (1988) 97.

[3] U. Edlund, A.Klarbring, Computer Methods in Applied Mechanics and Engineering, 96 (1992) 329.

[4] R. H. Andruet, D. A. Dillard, S. M. Holzer, Int. J. of Adhesion and Adhesives, 21 (2001) 17.

[5] J. P. M. Goncalves, M. F. S. F. Moura, P.M.S.T.Castro, Int. J. of Adhesion and Adhesives, 22 (2002) 357.

[6] L. Tong, X. Sun, Computational Mechanics, 30 (2003) 143.

[7] A. D. Crocombe, D. A. Bigwood, G. Richardson, 10 (3) (1990) 167.

[8] D. A. Bigwood, A. D.Crocombe, Int. J. of Adhesion and Adhesives, 10 (1) (1990).

[9] J. A. Harris, R. D. Adams, Int. J. Adhesion and Adhesives, 4 (2) (1984) 65.

[10] T. Carlberger, U. Stigh, Engng. Fracture Mech, 74(14) (2007) 2247.

[11] J. P. M. Goncalves, M. F. S. F.De Moura et al, Fatigue Fract. Engng Master Struct, 26(5) (2003) 479.

[12] H. Hadavinia, L. Kawashita, A. J. Kinloch, D. R. Moore, J. G. Williams, Engng. Fracture Mechanics, 73(16) (2006) 2324.

[13] P. Schmidt, U. Edlund, Int. J. for Num. Meth. In Engng, 1 (2005) 1.

[14] N. Valoroso, L. Champaney, Engng Fracture Mechanics, 73 (18) (2006) 2274.

[15] D. Castagnetti, E. Dragoni, Int. J. Adhes. and Adhes., 29 (2009) 125.

[16] L. Goglio et al., Int. J. Adhes. and Adhes, 28 (2008) 427.

[17] D. Castagnetti, A. Spaggiari, E. Dragoni, Proceedings of the 36th AIAS, Ischia (NA) (2007).

[18] Loctite - Hysol 9466, Technical Data Sheet, (Febbraio 2006).

[19] Loctite -7063, Technical Data Sheet, (Febbraio 2006).

[20] D. A. Bigwood, A. D.Crocombe, Int. J. Adhes. and Adhes, 9 (1989) 229.

[21] A. Pirondi, F. Moroni, ABAQUS Regional Users' Meeting, Milano (2008). 
[22] D. Castagnetti, , A. Spaggiari, , E. Dragoni, "Efficient Post-Elastic Analysis of Bonded Joints by Standard Finite Element Techniques", (2008, In press).

[23] ABAQUS 6.7, “Users' Manual”, HKS Inc. (2006).

[24] A. Pirondi, D. Fersini, E. Perotti, F. Moroni”, Atti del $19^{\circ}$ Congresso IGF, Milano (2007). 